\title{
Imaging of nonlinear and dynamic functional brain connectivity based on EEG recordings with the application on the diagnosis of Alzheimer's disease
}

\author{
Yifan Zhao*, Senior Member, IEEE, Yitian Zhao*, Pholpat Durongbhan, Liangyu Chen, Jiang \\ Liu, S. A. Billings, Panagiotis Zis, Zoe C. Unwin, Matteo De Marco, Annalena Venneri, Daniel \\ J. Blackburn, and Ptolemaios G. Sarrigiannis
}

\begin{abstract}
Since age is the most significant risk factor for the development of Alzheimer's disease (AD), it is important to understand the effect of normal ageing on brain network characteristics before we can accurately diagnose the condition based on information derived from resting state electroencephalogram (EEG) recordings, aiming to detect brain network disruption. This paper proposes a novel brain functional connectivity imaging method, particularly targeting the contribution of nonlinear dynamics of functional connectivity, on distinguishing participants with $A D$ from healthy controls (HC). We describe a parametric method established upon a Nonlinear Finite Impulse Response model, and a revised orthogonal least squares algorithm used to estimate the linear, nonlinear and combined connectivity between any two EEG channels without fitting a full model. This approach, where linear and non-linear interactions and their spatial distribution and dynamics can be estimated independently, offered us the means to dissect the dynamic brain network disruption in AD from a new perspective and to gain some insight into the dynamic behaviour of brain networks in two age groups (above and below 70 ) with normal cognitive function. Although linear and stationary connectivity dominates the classification contributions, quantitative results have demonstrated that nonlinear and dynamic connectivity can significantly improve the classification
\end{abstract}

Copyright (c) 2019 IEEE. Personal use of this material is permitted. However, permission to use this material for any other purposes must be obtained from the IEEE by sending a request to pubspermissions@ieee.org. The paper was submitted on 27/06/2019. We thank Neurocare for purchasing the EEG equipment used in this work. This is a summary of independent research supported by BRC and carried out at the National Institute for Health Research (NIHR) Sheffield Clinical Research Facility. The views expressed are those of the authors and not necessarily those of the BRC, NHS, the NIHR, or the Department of Health. This work was supported in part by the Liaoning Science and Technology Plan Project under Grant 20180550047, in part by the Shenyang Science and Technology Plan Project under Grant 18013-0-58, and in part by the Liaoning Provincial Department of Education Research Funding Project (QN2019010). This work was also supported in part by the Lloyd's Register Foundation under Grant number GAl100113, National Science Foundation Program of China (61601029), Zhejiang Provincial Natural Science Foundation (LZ19F010001). (Corresponding author: Liangyu Chen; ${ }^{*}$ Yifan Zhao and Yitian Zhao are co-first authors.) accuracy, barring the group of participants below the age of 70, for resting state EEG recorded during eyes open. The developed approach is generic and can be used as a powerful tool to examine brain network characteristics and disruption in a user friendly and systematic way.

Index Terms- Alzheimer's disease, dementia, visualisation, System identification, Machine learning

\section{Introduction}

$\mathrm{E}$ LECTROENCEPHALOGRAPHY (EEG) is commonly used in everyday clinical practice mainly for providing evidence for the diagnosis, classification and management of patients with epilepsy but also other various brain disorders (e.g. dementia). When compared with other methods that provide information about anatomical structures like magnetic resonance imaging (MRI), computerised tomography (CT) and functional MRI (fMRI), EEG offers ultra-high time resolution [1], which is critical to understand brain function. Synchronous networks form and dissipate in the range of 100-300ms which is thought to be the meaningful operational brain temporal scale [2]. EEG is economical, non-invasive, easy to administer and widely available in most hospitals. On the other hand, fMRI is costly, requires highly trained staff, a significant number of

Yifan. Zhao and P. Durongbhan are with the School of Aerospace, Transport and Manufacturing, Cranfield University, Cranfield, UK (email: yifan.zhao@cranfield.ac.uk; pholpatd@gmail.com).

L. Chen is with the Department of Neurosurgery, Shengjing Hospital of China Medical University, Shenyang, China (chenly@sj-hospital.org).

Yitian Zhao is with the Cixi Institute of Biomedical Engineering, Ningbo Institute of Industrial Technology, Chinese Academy of Sciences, Ningbo, China (yitian.zhao@nimte.ac.cn).

L. Jiang is with Department of Computer Science and Engineering, Southern University of Science and Technology, Shenzhen, China (liuj@sustech.edu.cn).

S. Billings is with the Department of Automatic Control and Systems Engineering, University of Sheffield, Sheffield, UK (s.billings@sheffield.ac.uk).

P. Zis, Z. Unwin, M. De Marco, A. Venneri, D. J. Blackburn and P. G. Sarrigiannis are with the Department of Neurosciences, Sheffield Teaching Hospitals, NHS Foundation Trust, Royal Hallamshire Hospital, Sheffield UK (takiszis@gmail.com;Zoe.Unwin@sth.nhs.uk; m.demarco@shef.ac.uk;a.venneri@shef.ac.uk;d.blackburn@shef.ac.u k;p.sarrigiannis@shef.ac.uk). 
patients experience symptoms of anxiety and claustrophobia, while it is also very sensitive to head movements. The latter, can be troublesome in patients with dementia where high levels of anxiety and restlessness are commonplace. Additionally, EEG directly measures brain electrical activity, thought to underpin cognitive functions, with far greater temporal resolution than fMRI which in return offers a very high spatial resolution, through measuring metabolic neuronal traces as a surrogate measure of their activity [3]. Empirical interpretation of the EEG is largely based on recognising abnormal frequencies in specific biological states (e.g. wakefulness versus sleep [4]), the spatio-temporal and morphological (e.g. sharp waves, spikes etc.) characteristics of paroxysmal [5] or persistent discharges [6], reactivity to external stimuli and activation procedures (like a period of hyperventilation [7] or intermittent photic stimulation [8]). Despite being useful in many instances, these practical approaches to interpret EEGs leave buried within the recordings very important dynamic and nonlinear interactions between various brain network anatomical constituents. There is significant evidence of this undetected information, for many neurological conditions, including epilepsy, neurodegenerative dementias, neuropsychiatric and movement disorders and normal cognitive paradigms [9]-[11]. Although EEG has been extensively studied over the last 30 years, there is a lack of systematic approaches to establish and analyse brain connectivity exclusively based on EEG recordings due to the complexity and the non-stationary behaviour of the signals; for example in the case of high levels of artefact, nonlinear dynamics and the fact that it is a typical ill-posed inverse problem.

There are three well-studied types of connection: anatomical, corresponding to white matter tracts between pairs of brain regions; functional, corresponding to magnitudes of temporal correlations in activity, and finally, effective connection representing direct or indirect causal influence of one region on another [12]. Most brain functions are performed not by single regions but by the combined coordinated activity of widely distributed brain networks. Several neurological and psychiatric disorders may be reflected in a breakdown of the ability of some brain regions to communicate effectively. This field was propelled forward when Watts and Strogatz [13] introduced the small-world network model, which described a network that provided regional specialisation with efficient global information transfer. Sporns et al. [14], Riviere et al. [15], Lohmann et al. [16], Stam et al. [17], and Lynall et al. [18] all focused on the structural topology of brain networks and their interactions by viewing the brain as a well-connected system, comprised of various regions that interrelate with each other to produce complex behaviours. In terms of effective connection, Kiebel et al. [19] presented a Dynamic Causal Modelling (DCM) for EEG and MEG, where a spatio-temporal neural mass mode was used to model the neuronal dynamics of each source. In combination with the Bayesian model comparison, it provides a useful way to test hypotheses about distributed processing in the brain. Studying brain functional connectivity is increasingly being recognised as an important approach for early diagnosis of many brain disorders (e.g.
Alzheimer's disease) [20]. From the system engineering point of view, the brain is a typical Complex System, which features many measurable components, interacting simultaneously and nonlinearly with each other and their environments at multiple levels [21]. The conversion of these observed measurements into knowledge about a physical object or system without a preknown function model has been one of the most important inverse problems, where nonlinear system identification is one of the most significant methodologies. Such a pure data-driven approach is attractive for brain studies because it allows less dependency on experience and knowledge of how brain functions. Although many parametric and non-parametric methods have been developed and applied to understand brain functional connectivity, these usually have the following limitations:

(1) Assumption that the connectivity is linear and stationary: Linear methods with assumptions about stationarity of the signals cannot sufficiently reveal and characterise hidden information of complex signals, commonly exhibiting dynamic and nonlinear behaviours. Brain network interactions are dynamic as phase synchronisation and phase scatter occur within the millisecond range (100-300ms) [2]. Additionally, nonlinearity is already introduced on the cellular level since the dynamical behaviour of individual neurones is governed by phenomena of integration, threshold, and saturation [22]. Transient associations, usually highly nonlinear, among different brain regions have been observed, for example in epileptic patients when moving from a normal state to a seizure [10], [11]. Exploring this otherwise undetectable information within EEG signals, far beyond the capabilities of commonly available methods, is crucial to better understand brain function and diagnose diseases.

(2) Dependence on rigorous assumptions and sufficiency of sampled data. For example, Granger causality [23] is one of the well-established methods to understand the interrelationship among EEG channels. However, this method and its extensions are model-based and require a known model structure before analysis and usually demand large number of samples to establish a full unbiased model [24]. This is far from straightforward when the underlying relationships in the system are nonlinear and dynamic and the measured observations are noisy because, unless a complete and full model which accounts for any potentially nonlinear noise effects is estimated, the results will be compromised. Nonparametric methods, such as entropy [25], tend to require larger data sets or averaging over many realizations to mitigate the effects of noise. The noise on the signals will usually be unknown prior to analysis but simple averaging methods will not work well if the noise is highly correlated and nonlinear, which may be expected if the relationships are also nonlinear.

It is well recognised that the integrity of dynamically interacting widely distributed brain networks, supported by widespread anatomical interconnections, is a prerequisite for normal brain function and that neurodegenerative conditions, to include the dementias, are associated with distinct patterns of brain network disruption [26]. Since age is considered the most significant risk factor for the development of $\mathrm{AD}$ [27], resting 
state EEG recordings have been increasingly used to study and define the effect of normal ageing on brain network characteristics [28] and to distinguish it from pathological ageing and specifically AD. A variety of electrophysiological features to include the power and distribution of specific frequency bands, functional connectivity and signal complexity has been applied to reveal the electrophysiological mechanisms underlying $\mathrm{AD}$ [29] which to our knowledge remain still not fully elucidated.

Revealing the spatial characteristics and the dynamic properties and type of disruption involving brain networks would be a step in the right direction, aiming to develop a diagnostic tool and a sensitive biomarker that can track disease progression. Oscillatory synchronisation between EEG recordings has been commonly estimated in previous studies with coherence [30], a linear method of measuring synchronisation although other methods of functional coupling, that allow detection of nonlinear interactions, like the synchronisation likelihood have been also implemented to study network disruption in dementia [31].

When correctly applied and interpreted within the context of a system neuroscience framework, the imaging and study of brain functional connectivity can be a very powerful tool that has the potential to revolutionise our understanding of brain degradation or dysfunction in a user friendly and systematic way. To achieve this level of understanding, this paper introduces a new approach to estimate and visualise the brain functional connectivity based on EEG signals, without fitting a full model, focusing on nonlinear and dynamic interactions. The proposed approach is applied to understand the differences in brain functional connectivity between Healthy Controls (HC) and AD participants, divided in two separate age groups. A supervised machine learning approach is then employed to investigate how the nonlinearity and dynamics of functional connectivity contribute to the classification of $\mathrm{AD}$ and $\mathrm{HC}$ subjects.

\section{MATERIALS AND METHODS}

\section{A. Case selection}

Participants were $\mathrm{HC}$ or patients diagnosed with $\mathrm{AD}$ who had detailed neuropsychology testing and structural and functional (fMRI) scans. All subjects were recruited from Sheffield Teaching Hospitals NHS Trust memory clinic, and HCs were enrolled through opportunity sampling and word of mouth over a period of a year (February 2015-16). Twenty HCs $(10<70 y$ old, 11 females, $10>70 y$ with a mean age of $67 y+/-S D$ of $12 y$ ) and $20 \mathrm{AD}$ cases (10 female, $16<70 \mathrm{y}$ and $4>70 \mathrm{y}$, mean age $64 \mathrm{y}+/-\mathrm{SD}$ of $8 \mathrm{y}$ ) were collected. Information regarding years of education, neuropsychology examinations and structural MRI findings are described in great detail in previously published work [9] and on Table A3 of Supplementary Material. The NINCDS-ADRDA criteria [32] were used to diagnose AD, based on a consensus taking into account clinical history, neurological examination, neuropsychological scores and neuroradiological findings. This project was approved by the Yorkshire and the Humber (Leeds West) Research Ethics
TABLE I

THE NUMBER OF SUBJECTS OF SIX STUDIED GROUPS

\begin{tabular}{ccc}
\hline \hline Group & AD & HC \\
\hline Below 70 EO & 16 & 10 \\
Above 70 EO & 4 & 10 \\
Below 70 EC & 16 & 10 \\
Above 70 EC & 4 & 10 \\
\hline \hline
\end{tabular}

Committee (reference number 14/YH/1070) and informed consent was provided by all participants.

\section{B. EEG recordings}

A modified 10/10 overlapping a 10/20 international system of electrode placement was adopted. All recordings were undertaken with the XLTEK 128-channel headbox (Optima Medical LTD) at a sampling rate of $2 \mathrm{~K} \mathrm{~Hz}$ (analogue low pass filter at $680 \mathrm{~Hz}$ ). An earlobe reference was used (jump cables were devised to combine the right and left earlobe electrodes; impedances where kept equal between sides). Thirty minutes, resting state, EEG recordings were acquired and distinct eyes open (EO) and eyes closed (EC) 5-minute epochs were obtained (throughout the recording period we encouraged all participants to rest and to try and keep their minds free of thought). They were readily prompted if their EEGs showed any signs of drowsiness.

Table I categorises patients in 4 groups, based on their age group, either below or above 70 . For each participant, data was collected for two separate physiological states: EO and EC. Time-locked video was recorded with the aforementioned XLTEK equipment (Optima Medical LTD). Bipolar derivations were used to revise all EEG data in this study and the following bipolar channels were available: F8-F4, F7-F3, F4-C4, F3-C3, F4-FZ, FZ-CZ, F3-FZ, T4-C4, T3-C3, C4-CZ, C3-CZ, CZ-PZ, C4-P4, C3-P3, T4-T6, T3-T5, P4-PZ, P3-PZ, T6-O2, T5-O1, P4-O2, P3-O1, O2-O1. Subsequently, both for EO and EC, 12 seconds in duration, artefact free epochs were selected for analysis. To avoid bias (i.e. empirical data interpretation and selection), the first $12 \mathrm{~s}$ EO/EC epochs, isolated from each of the 40 participants with Spike 2 (version 8) software, were used for data-processing and export. Slow EEG frequency artefacts, like those generated by eye blinking and movements, in the low delta and below ranges, were attenuated by applying to all data a time constant, $\tau=0.08 \mathrm{~s}$ (high pass filter at $2 \mathrm{~Hz}$ ). A notch filter was also applied to all channels. For this paper, the middle $4 \mathrm{~s}$ section of this $12 \mathrm{~s}$ data was selected for further connectivity analysis to avoid the boundary issue.

\section{Nonlinear functional connectivity estimation}

This paper proposes a revised orthogonal least squares (ROLS) algorithm to measure the connectivity between two EEG channels. OLS is an approach that has been used in nonlinear system identification where it searches through all possible candidate model terms to select the most effective ones to build the model [33]. The significance of each selected model term is measured by the Error Reduction Ratio (ERR) index which indicates how much of the change in the system response, in percentage, can be accounted for by including the 
relevant model terms. This capability allows the computing of the contribution of linear and nonlinear terms independently without fitting the full nonlinear model, where parameter estimation and model validation are required.

Considering a single input and single output (SISO) system with input time series $\{u(1), u(2), \ldots, u(M)\}$ and time-varying output time series $\{y(1), y(2), \ldots, y(M)\}\}$, where $M$ denotes the number of data points. To quantify and track the correlation of the input to the output, this paper uses a Nonlinear Finite Impulse Response (NFIR) model, also known as the Volterra Nonlinear Regressive with eXogenous (VNRX) Inputs model, to represent a SISO system. It can be expressed as

$$
y(t)=f\left(u^{\left[t-n_{u}\right]}\right)+\varepsilon(t),
$$

where $t(t=1,2, \ldots)$ is a time index, $f$ is an unknown linear or nonlinear mapping which links the system output $y$ to the inputs; $\varepsilon(t)$ denotes the model residual. The symbol $u^{\left[t-n_{u}\right]}$ denotes the current and past information of the input $u$, which can be expanded as

$$
u^{\left[t-\mathrm{n}_{u}\right]}=\left\{\bigcup_{j=0}^{n_{u}} u(t-j)\right\},
$$

where $n_{u}$ is the maximal temporal lag to be considered for the input $u$.

If the system is time-invariant, a commonly employed implementation to specify the function $f$ in Eq. (1) is a polynomial function, which can be expressed as

$$
y=\theta_{0}+\sum_{m=1}^{N} \theta_{m} p_{m}+\varepsilon,
$$

where $p_{m}$ is the $\mathrm{m}^{\text {th }}$ model term selected from a candidate term set constructed from all input vectors. Note that $p_{m}$, in general, can be linear or nonlinear. The constant $\theta_{m}$ is the coefficient of each term; $N$ is the total number of model terms.

If the model order is set as $q$, the candidate term set, denoted by $C$, can be expressed

$$
C=C_{1} \cup C_{2} \cup \ldots \cup C_{l} \cup \ldots \cup C_{q},
$$

where $C_{1}$ is the linear term set, expressed as

$$
C_{1}=\cup_{j=0}^{n_{u}} u(t-j)
$$

and $C_{2}$ is the $2^{\text {nd }}$ order nonlinear term set, expressed as

$$
C_{2}=\cup_{a_{1}=0}^{n_{u}} \cup_{a_{2}=a_{1}}^{n_{u}} u\left(t-a_{1}\right) u\left(t-a_{2}\right)
$$

and $C_{l}$ is the $l^{\text {th }}$ order nonlinear term set, expressed as

$$
C_{l}=\cup_{a_{1}=0}^{n_{u}} \bigcup_{a_{2}=a_{1}}^{n_{u}} \ldots \cup_{a_{l}=a_{l-1}}^{n_{u}} \prod_{i=1}^{l} u\left(t-a_{\mathrm{i}}\right) .
$$

If the inputs and output of a system are observable, the model represented by Eq. (3) can then be identified based on the principle of least square errors. Equation (3) is re-written as

$$
Y=P \Theta,
$$

where

$$
Y=\left[\begin{array}{c}
y(1) \\
y(2) \\
\vdots \\
y(M)
\end{array}\right], P=\left[\begin{array}{c}
P^{T}(1) \\
P^{T}(2) \\
\vdots \\
P^{T}(M)
\end{array}\right], \Theta=\left[\begin{array}{c}
\theta(1) \\
\theta(2) \\
\vdots \\
\theta(M)
\end{array}\right]
$$

and $P^{T}(k)=\left(p_{1}(k), p_{2}(k), \ldots, p_{N}(k)\right) . p_{1}, p_{2, \ldots,} p_{N}$ are the model terms selected from the candidate term set, written as Eq. (4). Matrix $P$ can be decomposed as $P=W \times A$ where

$$
W=\left[\begin{array}{cccc}
w_{1}(1) & w_{2}(1) & \ldots & w_{N}(1) \\
w_{1}(2) & w_{2}(2) & \ldots & w_{N}(2) \\
\vdots & \ddots & \ddots & \vdots \\
w_{1}(M) & w_{2}(M) & \ldots & w_{N}(M)
\end{array}\right]
$$

and $A=\left\{a_{i j}\right\}$ is an upper triangular matrix with unity diagonal elements. Equation (8) is then rewritten as

$$
Y=W G,
$$

where $G=A \Theta=\left[\begin{array}{llll}g_{1} & g_{2} & \cdots & g_{N}\end{array}\right]^{T}$. Equation (11) is now ready to represent the relation between $\mathrm{Y}$ and $\mathrm{G}$.

The importance of each model term to the variation of the system output is then estimated. Initially, set values $a_{i j}=0$ for $i \neq j$ ( $A$ then becomes an identity matrix), so $w_{1}(k)=p_{1}(k)$, and calculate $g_{1}$ as

$$
g_{1}=\frac{\sum_{k=1}^{M} w_{1}(k) y(k)}{\sum_{k=1}^{M} w_{1}^{2}(k)} .
$$

For $j=2,3, \ldots, M$, set $a_{j j}=1$ and then calculate

$$
a_{i j}=\frac{\sum_{k=1}^{M} w_{i}(k) p_{j}(k)}{\sum_{k=1}^{M} w_{i}^{2}(k)}
$$

where $i=1,2, \ldots, j-1$. Next, the algorithm calculates

$$
w_{j}(k)=p_{j}(k)-\sum_{i=1}^{j-1} a_{i j} w_{i}(k)
$$

and

$$
g_{1}=\frac{\sum_{k=1}^{M} w_{j}(k) y(k)}{\sum_{k=1}^{M} w_{j}^{2}(k)} .
$$

The ERR value for each term $p_{i}$ is finally defined as

$$
E R R_{i}=\frac{\hat{g}_{i}{ }^{2} \sum_{k=1}^{H} x_{i}{ }^{2}(k)}{\sum_{k=1}^{H} y^{2}(k)} .
$$

Values of ERR range from $0 \%$ to $100 \%$. The larger the value of ERR, the higher the dependence between this term and the output. To stop the search procedure and determine the number of significant terms $N$, a criterion called Penalised Error-toSignal Ratio (PESR) is used [34]. It can be written as

$$
\operatorname{PESR}_{m}=\frac{1}{\left(1-\frac{\lambda m}{H}\right)^{2}}\left(1-\sum_{i=1}^{m} E R R_{i}\right)
$$

This criterion is introduced to monitor the search procedure, where $m$ denotes the index of the selected terms. The search procedure stops when $P E S R_{m}$ achieves a local minimum. In this paper, the value of $\lambda$ was chosen as 8 .

To calculate the contribution of the input to the output, the sum of ERR of all selected terms, denoted by $S E R R$, is calculated by

$$
S E R R=\sum_{i=1}^{N} E R R_{i}
$$

Note $N$ is the number of the selected terms, not the number of total candidate terms. The value of $\operatorname{SERR}(0 \leq S E R R \leq 1)$ describes the percentage explained by the identified model to 
the system output. If the considered inputs can fully explain the variation of the system output, the value of $S E R R$ is equal to $100 \%$.

The linearity of connectivity is represented by the sum of ERR of the terms that are linear, and it can be computed by

$$
\operatorname{ERR}_{L}(y, u)=\frac{\left.\sum_{b=1}^{N}\left(E R R_{b}\right) \mid p_{b} \in C_{1}\right)}{S E R R}
$$

The nonlinearity of connectivity is represented by the sum of ERR of the terms that are nonlinear, and it can be computed by

$$
E R R_{N}(y, u)=\frac{\sum_{b=1}^{N}\left(E R R_{b} \mid p_{b} \notin C_{1}\right)}{S E R R} .
$$

Considering the estimated $E R R_{L}$ and $E R R_{N}$ being the measurement of bidirectional functional connectivity between two signals, with 23 channels used in this study, there are 253 possible combinations $\left(C_{2}^{23}\right)$ when any 2 channels are paired together. These were organised in a pairwise manner by taking the first channel in the list (F8-F4) and pairing it with every other channel according to their order (F8-F4:F7-F3, F8-F4:F4$\mathrm{C} 4, \mathrm{~F} 8-\mathrm{F} 4: \mathrm{F} 3-\mathrm{C} 3$...). The process was subsequently repeated for all other channels to the end of the list. However, since each channel is bipolar in nature, any pair with common electrode locations (such as F8-F4 and F4-C4) is neglected as this could lead to misleadingly high false correlation between the pair. There are 46 channel pairs that have this characteristic. A total of 207 channel pairs is therefore analysed in this paper.

\section{Functional connectivity dynamics estimation}

Dynamic Range of Connectivity (DRC) is introduced in this research to describe the dynamics of functional connectivity across multiple epochs (5 mini-epochs per participant both for EC and EO states). This was estimated both for linear and nonlinear connectivity in every EEG recording. DRC is based on the more commonly known "dynamic range" which is defined as the ratio between the largest and smallest values of that signal. DRC in this study is also based upon this definition. However, given the nature of the ERR estimates used in this study, some elements in the series of 5 values from 5 epochs can have 0 and would render the value of that series' DRC infinitely large. To address this issue, in the case where the smallest element is 0 , while the largest value is not, DRC is calculated using the second-smallest non-zero element instead. For the case in which the maximum is the only non-zero element, DRC is defined as 1 to reflect the fact that the minimum and maximum of non-zero values are the same. However, for the case in which the series consists entirely of 0 , DRC is defined as 0 to represent the lack of dynamics for that series. Transient associations among different brain regions have been reported [10], [11] for various brain-related disorder, but have not been fully explored. This paper investigates the difference of DRC within both linear and nonlinear associations among all EEG channels in HC and AD participants.

\section{E. Connectivity Visualisation}

A revised Circular Graph (RCG) is introduced in this paper to visualise the ERR based connectivity estimates. The visualisation functionality was developed based on the original

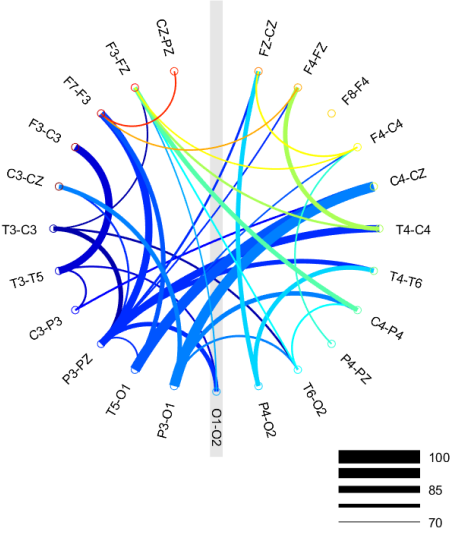

Fig. 1. An example of the introduced Revised Circular Graph for visualising the brain functional connectivity with the legend in the rightbottom corner. The user-specified threshold and maximum value for this plot are 70 and 100 respectively.

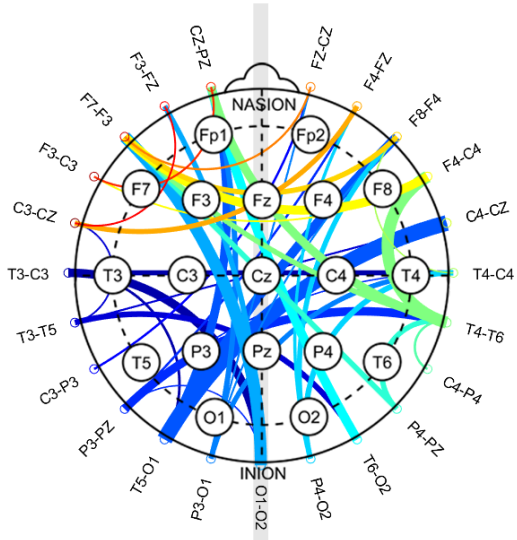

Fig. 2. The proposed RCG plot overlaid with EEG electrode locations to highlight the real electrode locations and their corresponding locations in the plot.

code written by Kassebaum [35] and was modified to include additional features to fit this research. The visualisation function draws a circular plot where the channel names are placed on the circumference of a circle. The connectivity values between channels are drawn as lines connecting the two channels, with its width representing the strength of the connection between two channels. The RCG also plots a legend for the plot containing 5 lines of differing width, with their corresponding values in the lower right corner, as can be seen in Fig. 1. Plotting the values for every channel pair would overwhelm the readers with too much information at once. Thus, RCG performs a thresholding operation prior to plotting, with only values higher than a specified threshold being plotted for visualisation. The threshold is selected by looking at the max, mean, and STD of the values in all datasets within the same plot.

To further improve the interpretability of the plot, the location of the channels is based on the location of the electrode channels used in the datasets. The central channels (CZ-PZ, FZ$\mathrm{CZ}$ ), since plotting them directly in the middle of the circle would render the plot unreadable, are placed instead together at the top of the plot. The rest of the channels are, then, placed in order from the frontal to the occipital areas. A grey middle line 
is also drawn to clearly mark the left-right hemisphere. To better illustrate this paradigm, an example plot, overlaid with electrode locations is shown in Fig. 2.

\section{F. Classification using Machine Learning}

The proposed classification using machine learning approaches based on features from ERR connectivity values, was developed as an add-on to the previously proposed dementia classification framework [36]. As already fine-tuned and implemented in the original framework, K-Nearest Neighbour $(\mathrm{KNN})$ classification method where $\mathrm{K}=1$ with 10 fold cross-validation was used in this paper. Each value of classification accuracy in this paper was calculated through averaging 50 iterations of classifications.

To capture the dynamics of functional connectivity of the data, the following procedures were done. For each dataset, the $4 \mathrm{~s}$ EEG record was equally divided into 5 mini-epochs, each of which is with a duration of $0.8 \mathrm{~s}$. For each epoch, the linear and nonlinear ERR values of each pair of electrodes, $E R R_{L}$ and $E R R_{N}$, were calculated based on Eq. (19) and Eq. (20) respectively. The combined ERR values, $S E R R=E R R_{L}+$ $E R R_{N}$, are also calculated. Therefore, 15 features were extracted (5ERR $R_{L}$ values, $5 E R R_{L}$ values, and $5 S E R R$ values) from each bipolar pair.

Arithmetic mean (Mean) and root-mean-square (RMS) of three types of connectivity for all five mini-epochs are used as two final features representing the average magnitude of functional connectivity for classification. In addition, statistical range (Range) and DRC are also produced for the same data, as two further final features for classification, representing the dynamics of functional connectivity. It should be noted that, in this paper, the number of features is kept consistently the same during comparisons to ensure unbiased outcomes. The following two analyses were conducted using the aforementioned features:

Linear vs Nonlinear Component: The objective of this analysis is to inspect and quantify the impact of nonlinear connection on the classification accuracy. This impact was determined by comparison of classification performance of the linear features and the combined features, rather than the nonlinear features only (there are many elements with zero value in nonlinear features that affect the classification). Any improvement of performance using the combined features is contributed by the nonlinear component. Both Mean and RMS were used as features.

Mean Magnitude vs DRC: It is well accepted that brain connections are dynamic and that functional connectivity is highly temporal in nature [37]. This analysis aims to investigate if the consideration of dynamics of connection can improve the performance of classification of $\mathrm{AD}$ and $\mathrm{HC}$ in the context of both linear and nonlinear components. It should be noted that the nonlinear connectivity is used directly to produce our estimates, as the elements with zero value have been neglected during DRC calculation.

\section{Results}

\section{A. Functional connectivity magnitude in $A D$ and $H C$ Subjects}

In this study, strength of functional connectivity, represented by the linear and nonlinear ERR components, was obtained by averaging the 5 ERR values of a specified component (linear, nonlinear or combined) of each channel pair for each subject. These mean magnitudes were then averaged over all subjects in that group according to their label $(\mathrm{AD} / \mathrm{HC})$ to produce an average mean magnitude (AMM) value for each channel pair for a specific label. The resulting AMM values for linear and nonlinear components are plotted in Fig. 3 and Fig. 4 respectively.

In Fig. 3, the linear AMM shows a similar pattern in terms of significant connections and corresponding strength for $\mathrm{HC}$ subjects among all eye states and age groups, except for the Below 70 EC group where there is high connectivity strength among the parietal, temporal, and occipital areas. Contrary to the $\mathrm{HC}$ subjects, the linear AMM of AD subjects shows no consistent patterns among age groups nor eye states. The AD subjects in below $70 \mathrm{EO}$ and above $70 \mathrm{EC}$ show visible increase in the number of significant connections and strength for linear components, while the AD subjects in below $70 \mathrm{EC}$ and above 70 EO data show similar patterns to HC subjects (Fig. 3).

The nonlinear AMM, plotted in Fig. 4, shows that in the EO and EC data for the below 70 cohorts, HC subjects display slightly more nonlinear connectivity among more channel pairs than their AD counterpart, mainly for the EO state. However, for the above $70 \mathrm{EO}$ and EC states, the reverse can be observed with AD subjects showing significantly higher nonlinear connectivity strength than HC subjects in nearly all channel pairs. Even though there is an increase in the average magnitude of nonlinear connectivity both for $\mathrm{AD}$ and $\mathrm{HC}$ subjects in the elderly group compared to the below 70 groups, the most significant increase is observed in the older $\mathrm{AD}$ group during EO.

Furthermore, similar as in Fig. 3 for the above 70 HC cohort, it is shown in Fig. 4 that the nonlinear connectivity strength in HC subjects remains relatively consistent throughout all eye states, with only a small drop in the below 70 EC group. From both figures, it can be inferred that for the older participants, HC subjects display relatively consistent linear connectivity strength, for EO and EC states but nonlinear estimates appear fairly consistent both for younger and older HC participants for both eye states. This contrasts with the significant changes seen for the linear levels of connectivity strength between EO and $\mathrm{EC}$ states in $\mathrm{AD}$, involving both the younger and older age group, albeit moving in opposite directions (i.e. higher for EO for those $<70$ and higher for EC for $>70$ ). On the other hand, the nonlinear estimates for the AD, show consistent findings for EO and EC states for each age group but striking differences in strength of connectivity for the below and above 70 cohorts, the latter showing brain networks engaging in much higher levels of widespread nonlinear synchronisation. 
Dataset

AD

Below 70 EO

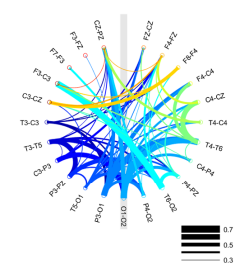

Below 70 EC

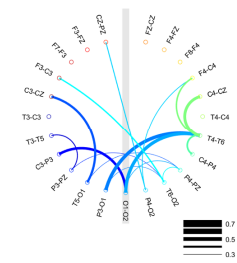

Above 70 EO

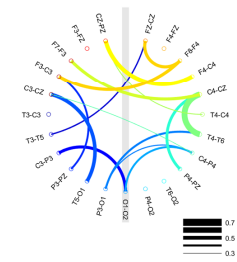

Above 70 EC

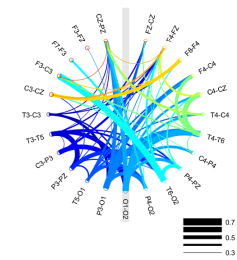

Fig. 3. The RCG plots of the AMM values of the linear connectivity for all subjects in a group. The threshold for all plots is set as 0.3 .

\section{B. Functional connectivity dynamics in $A D$ and $H C$ Subjects}

In a similar manner as the last section, the functional connectivity dynamics of both linear and nonlinear ERR components, represented by the DRC of each channel pair, was calculated for each subject and each channel pair. By averaging DRC for a particular channel pair but over all subjects with the same label $(\mathrm{AD} / \mathrm{HC})$ separately for $\mathrm{EO}$ and $\mathrm{EC}$ states, the resulting average DRC for each channel pair was obtained and plotted in Fig. 5 and Fig. 6 for linear and nonlinear associations, respectively.

Inspection of Fig. 5 shows that the average DRC of linear connectivity does not differ significantly between $\mathrm{AD}$ and $\mathrm{HC}$ subjects between EO and EC states, more obvious for those above 70. However, significantly higher nonlinear dynamics can be observed in Fig. 6, for the above $70 \mathrm{AD}$ cohort, in comparison to the $\mathrm{HC}$ group both for $\mathrm{EO}$ and $\mathrm{EC}$ states. For the below 70 groups, HCs show higher level and more widespread nonlinear dynamic variability for the EO state. It is also worth noting that many more electrode pairs exhibit dynamic nonlinear connectivity fluctuations in the EO versus the EC state.

\section{Classification of $A D$ and $H C$ subjects}

An overview of the average classification accuracy of the top 10 channel pairs with the best classification performance for linear, nonlinear, and combined connectivity is displayed in

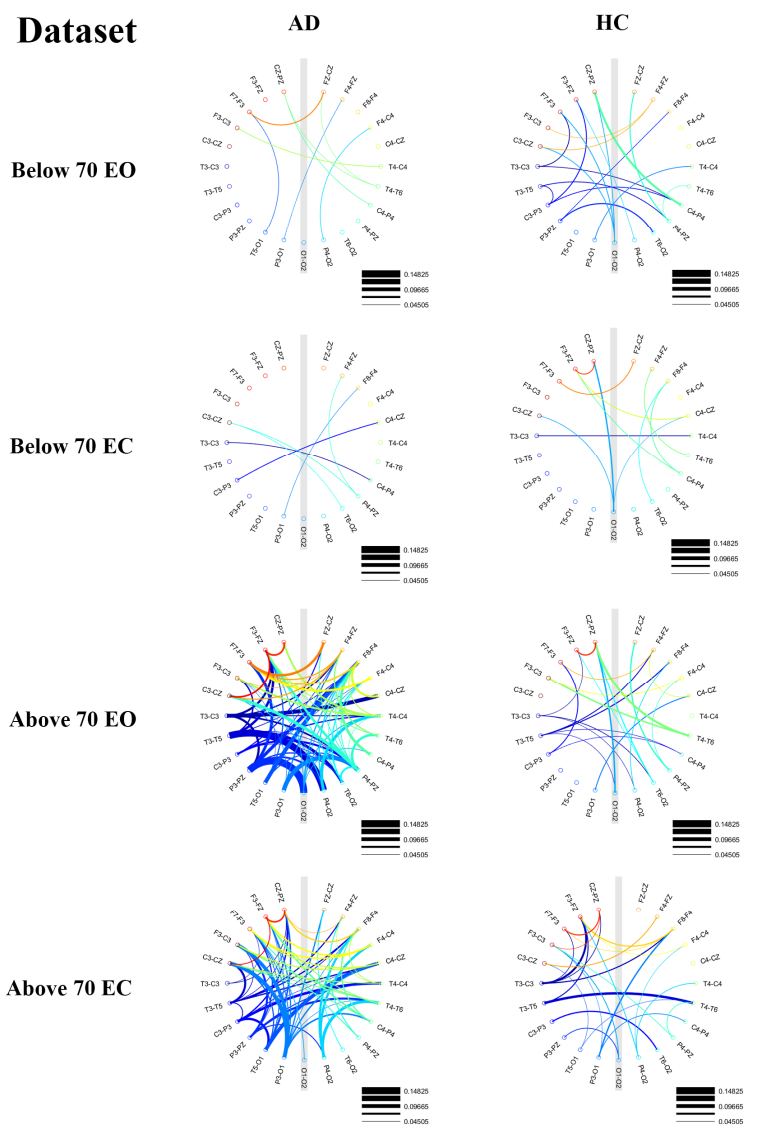

Fig. 4. The RCG plots of the AMM values the nonlinear connectivity for all subjects in a group. The threshold for all plots is set as 0.04505 .

TABLE II

THE AVERAGE CLASSIFICATION ACCURACY AMONG THE TOP 10 CHANNEL PAIRS WITH HIGHEST CLASSIFICATION ACCURACY IN EACH DATA SET FOR MEAN AND RMS FEATURES. (IN \%, MEAN(SD))

\begin{tabular}{cccc}
\hline \hline Group & Linear & $\begin{array}{c}\text { Mean \& RMS } \\
\text { Nonlinear }\end{array}$ & Combined \\
\hline Below 70 EO & $78.42(3.44)$ & $78.77(3.58)$ & $80.33(3.26)$ \\
Above 70 EO & $83.54(3.28)$ & $86.71(5.57)$ & $86.56(4.04)$ \\
Below 70 EC & $72.00(2.11)$ & $74.35(2.66)$ & $74.5(4.38)$ \\
Above 70 EC & $88.54(2.88)$ & $80.98(1.05)$ & $90.5(5.34)$ \\
\hline \hline
\end{tabular}

Table II. It can be clearly observed that the linear connectivity plays the dominant role in classification. It also shows that, for the magnitude features (Mean and RMS), the nonlinear connectivity in isolation does not provide a clear change in classification accuracy compared to results from the linear connectivity, which may be caused by the large amount of zero values.

However, results from the combined connectivity provide the best classification accuracy for all groups (up to $90 \%$ average accuracy). It should be noted that the number of features used for all three tests was the same to ensure unbiased results. It can be inferred that adding nonlinear components into the algorithm has an impact on the overall average classification accuracy, although the linear component has the dominant contribution.

To elaborate on the impact of nonlinear components on the classification accuracy, two sets of test were conducted: the first one used Mean and RMS features of linear connectivity while 


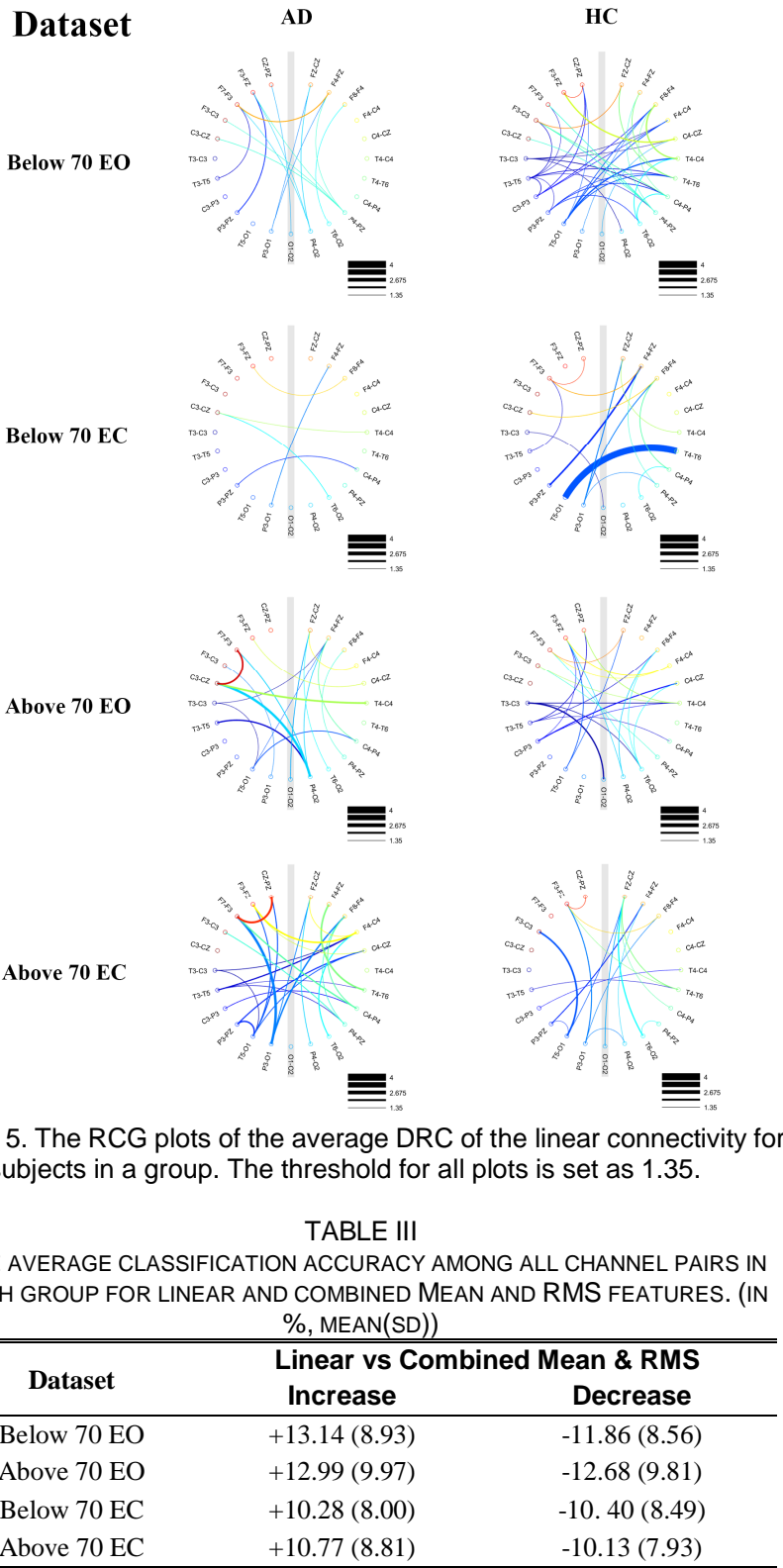

the second, combined linear and nonlinear connectivity. The difference in classification accuracy for each channel pair between the two tests was determined. They were grouped according to whether the classification accuracy for the combined group had increased or decreased from their linear counterpart and the results are shown in Table III. Table III conclusively shows that when nonlinear components were considered in addition to the linear estimates, the classification power of the features is impacted both positively and negatively (>8\% average change for all groups). However, this change varies across different eye states, with EO seeing slightly more changes to the average classification accuracy $(>11 \%$ average change) than eyes closed state ( $>10 \%$ average change).

Further analysis of individual changes in accuracy for each channel pair is illustrated in Fig. 7 where the minimum threshold for all plots is $20 \%$ and the maximum value is $50 \%$. For the EO state, adding the nonlinear components induces

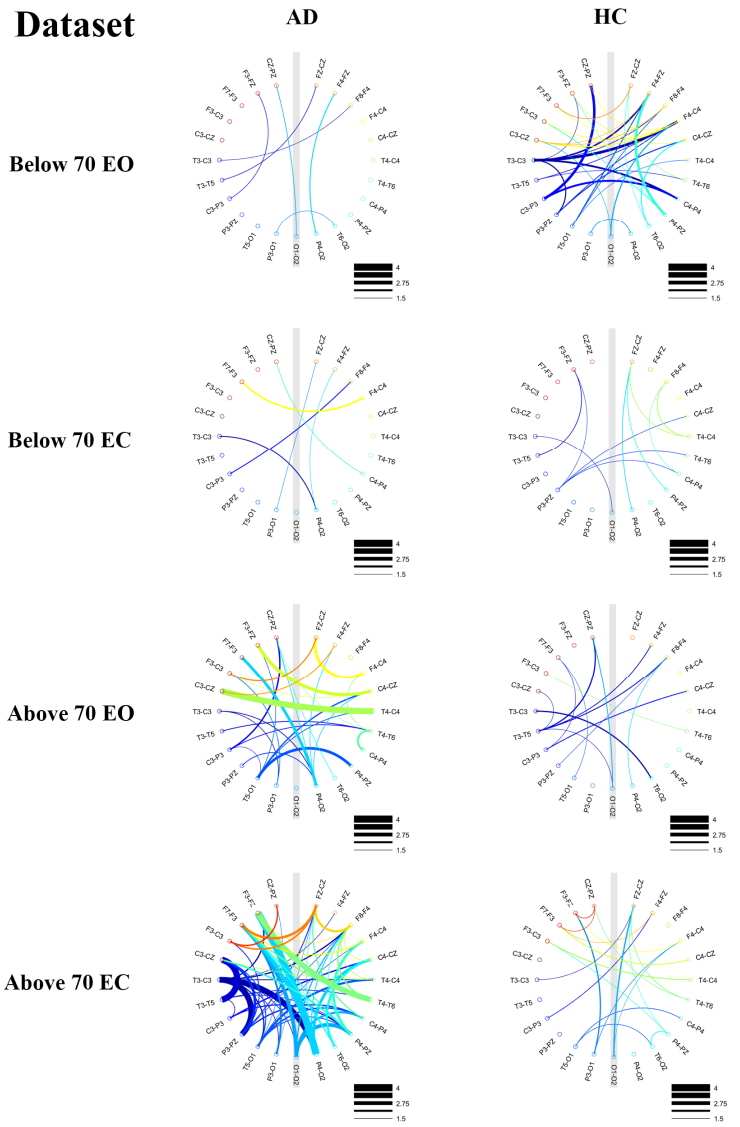

Fig. 6. The RCG plots of the average DRC of the nonlinear connectivity for all subjects in a group. The threshold for all plots is set as 1.5 .

stronger changes in the classification accuracy for channels pairs which are in different hemispheres. In contrast, strong changes for both age groups in EC state tend to occur to channel pairs in the same, left or right hemisphere. Additionally, the results for EC state in Fig. 7 shows that it is in line with the results in Table III where weaker changes can be observed in both age groups.

Focusing in isolation at the rate of change in classification accuracy (Fig. 7) reveals which areas/electrode pairs are influenced by the inclusion of nonlinear connectivity, but it does not provide insight into their actual classification accuracy values after those changes. The top 5 channel pairs that have the best classification accuracy in terms of linear and combined connectivity are listed in Table IV. The results suggest that the best performing classification accuracies for the combined features are generally better than their linear counterpart. For the below 70 EO group, a slight increase (up to $2 \%$ ) of accuracy is observed; while for the below 70 EC group, an up to $8 \%$ boost can be observed. Using the combined features, the above 70 cohorts obtain classification accuracy of up to $100 \%$ for 2 channel pairs in the EC state and up to $93 \%$ in the EO state.

Addressing the second analysis in Section 2.6, Table 5 shows a comparison of classification performance between dynamics and mean magnitude features for both linear and nonlinear connectivity. For the linear connectivity, no significant difference in classification was observed between mean magnitude and dynamics. However, for the nonlinear 


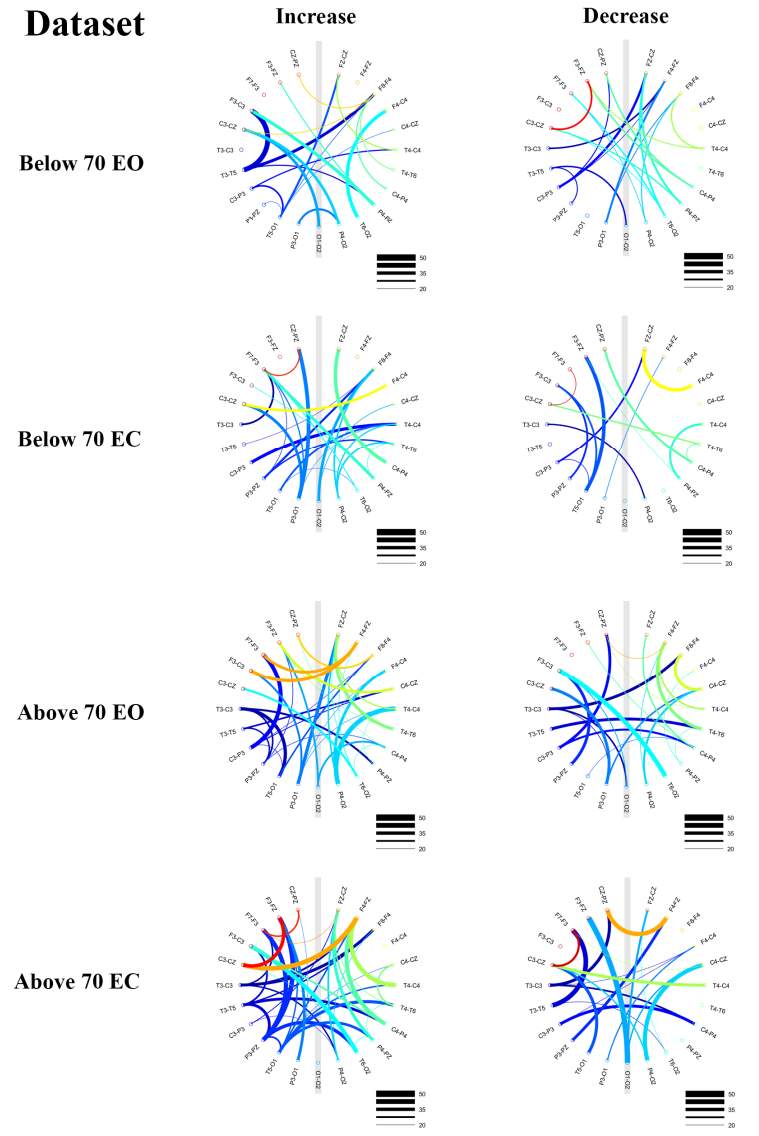

Fig. 7. The RCG plots of the change in classification accuracy from models trained with Mean and RMS of linear connectivity to models trained with Mean and RMS of combined connectivity.

connectivity, the dynamics features perform better than mean magnitude connectivity (up to $7 \%$ better) for all groups except for the above $70 \mathrm{EO}$ group.

\section{Discussions}

The high levels of linear synchronisation estimated with the AMM (Fig. 3) for EO, for the younger AD cohort, are a clear expression of network dysfunction, as it is common knowledge that healthy subjects typically exhibit a desynchronised EEG during periods of eyes open. This can be easily seen by comparing the age matched RCG findings for the $\mathrm{HCs}$ in the same figure; the differences between $\mathrm{AD}$ and $\mathrm{HC}$ are prominent. Noticeably, the DRC estimates, expressing the dynamic changes in the strength of synchronisation over time (Fig. 5) show, for the EO state for the same age group, very little variation in AD. Therefore, widely distributed networks appear "locked" in high levels of linear synchronisation. This observation confirms the validity of the concept suggesting a balanced and temporally precise pattern of synchronisation and desynchronisation is pertinent to cognitive functions [38]. On the other hand, in comparison to the $\mathrm{AD}$ group, the nonlinear synchronisation AMM estimates are more widespread in HCs for EO state below the age of 70 (Fig. 4). More importantly, the dynamic variability of nonlinear interactions, as expressed by the DRC, is also much higher and widely distributed for HCs
TABLE IV

THE TOP 5 CHANNEL PAIRS AND ITS CLASSIFICATION ACCURACY FOR EACH GROUP USING MEAN AND RMS FEATURES OF LINEAR AND COMBINED CONNECTIVITY. (IN \%, *ACC DENOTES ACCURACY)

\begin{tabular}{|c|c|c|c|c|c|c|c|}
\hline \multicolumn{4}{|c|}{ Below 70 EO } & \multicolumn{4}{|c|}{ Below 70 EC } \\
\hline \multicolumn{2}{|c|}{ Linear } & \multicolumn{2}{|c|}{ Combined } & \multicolumn{2}{|c|}{ Linear } & \multicolumn{2}{|c|}{ Combined } \\
\hline Pair & Acc & Pair & Acc & Pair & Acc & Pair & Acc \\
\hline $\begin{array}{l}\text { T3-T5: } \\
\text { P4-PZ }\end{array}$ & 85.19 & $\begin{array}{l}\text { F7-F3: } \\
\text { T6-O2 }\end{array}$ & 85.23 & $\begin{array}{l}\text { C4-CZ: } \\
\text { O1-O2 }\end{array}$ & 76.92 & $\begin{array}{l}\text { F4-C4: } \\
\text { P4-PZ }\end{array}$ & 85.23 \\
\hline $\begin{array}{l}\text { T4-C4: } \\
\text { P3-O1 }\end{array}$ & 81.54 & $\begin{array}{l}\text { C3-P3: } \\
\text { P4-PZ }\end{array}$ & 84.69 & $\begin{array}{l}\mathrm{F} 8-\mathrm{F} 4: \\
\mathrm{O} 1-\mathrm{O} 2\end{array}$ & 74.42 & $\begin{array}{l}\text { F4-FZ: } \\
\text { T5-O1 }\end{array}$ & 77.62 \\
\hline $\begin{array}{l}\text { CZ-PZ: } \\
\text { P3-O1 }\end{array}$ & 81.15 & $\begin{array}{l}\text { F4-FZ: } \\
\text { T4-C4 }\end{array}$ & 84.15 & $\begin{array}{l}\text { C4-P4: } \\
\text { C3-P3 }\end{array}$ & 72.88 & $\begin{array}{r}\text { FZ-CZ: } \\
\text { T4-C4 }\end{array}$ & 77.38 \\
\hline $\begin{array}{l}\text { F7-F3: } \\
\text { C4-CZ }\end{array}$ & 80.58 & $\begin{array}{l}\text { T3-C3: } \\
\text { P3-PZ }\end{array}$ & 81.00 & $\begin{array}{l}\text { P4-PZ: } \\
\text { O1-O2 }\end{array}$ & 72.50 & $\begin{array}{l}\text { T4-T6: } \\
\text { P4-PZ }\end{array}$ & 74.15 \\
\hline $\begin{array}{l}\text { C3-P3: } \\
\text { P4-PZ }\end{array}$ & 78.85 & $\begin{array}{l}\text { C3-P3: } \\
\text { T3-T5 }\end{array}$ & 80.38 & $\begin{array}{l}\text { F8-F4: } \\
\text { C3-P3 }\end{array}$ & 71.15 & $\begin{array}{l}\text { T4-C4: } \\
\text { C3-CZ }\end{array}$ & 73.54 \\
\hline \multicolumn{4}{|c|}{ Above 70 EO } & \multicolumn{4}{|c|}{ Above 70 EC } \\
\hline \multicolumn{2}{|c|}{ Linear } & \multicolumn{2}{|c|}{ Combined } & \multicolumn{2}{|c|}{ Linear } & \multicolumn{2}{|c|}{ Combined } \\
\hline Pair & Acc & Pair & Acc & Pair & Acc & Pair & Acc \\
\hline $\begin{array}{l}\text { FZ-CZ: } \\
\text { T3-T5 }\end{array}$ & 86.79 & $\begin{array}{l}\text { F7-F3: } \\
\text { FZ-CZ }\end{array}$ & 93.14 & $\begin{array}{l}\text { F8-F4: } \\
\text { C4-CZ }\end{array}$ & 92.86 & $\begin{array}{l}\text { C3-CZ: } \\
\text { O1-O2 }\end{array}$ & 100.00 \\
\hline $\begin{array}{l}\text { F4-FZ: } \\
\text { O1-O2 }\end{array}$ & 86.79 & $\begin{array}{c}\text { C4-CZ: } \\
\text { T3-T5 }\end{array}$ & 92.86 & $\begin{array}{l}\text { F3-C3: } \\
\text { P4-O2 }\end{array}$ & 92.86 & $\begin{array}{l}\text { F4-FZ: } \\
\text { P4-PZ }\end{array}$ & 100.00 \\
\hline $\begin{array}{l}\text { T4-C4: } \\
\text { O1-O2 }\end{array}$ & 86.07 & $\begin{array}{l}\text { C3-CZ: } \\
\text { P3-O1 }\end{array}$ & 88.00 & $\begin{array}{l}\text { F4-FZ: } \\
\text { T6-O2 }\end{array}$ & 92.86 & $\begin{array}{l}\text { F4-FZ: } \\
\text { T6-O2 }\end{array}$ & 92.86 \\
\hline $\begin{array}{l}\text { P3-PZ: } \\
\text { O1-O2 }\end{array}$ & 86.07 & $\begin{array}{l}\text { T3-C3: } \\
\text { C4-CZ }\end{array}$ & 87.14 & $\begin{array}{l}\text { F4-FZ: } \\
\text { C4-P4 }\end{array}$ & 87.86 & $\begin{array}{l}\text { F3-FZ: } \\
\text { P4-O2 }\end{array}$ & 92.86 \\
\hline $\begin{array}{l}\text { F8-F4: } \\
\text { F3-C3 }\end{array}$ & 85.71 & $\begin{array}{l}\text { F8-F4: } \\
\text { C4-P4 }\end{array}$ & 86.14 & $\begin{array}{l}\text { F8-F4: } \\
\text { C3-P3 }\end{array}$ & 87.14 & $\begin{array}{l}\mathrm{F} 3-\mathrm{C} 3: \\
\text { T6-O2 }\end{array}$ & 86.86 \\
\hline
\end{tabular}

for the same age group during EO. This suggests that a degree of nonlinear dynamic connectivity characterises brain network function of the younger group HC participants but there is no difference between EO and EC for the older cohort.

It is of interest that on work we have previously undertaken on an entirely different neurological condition, a paroxysmal epileptic disorder, childhood absence epilepsy, significant dynamic changes in linear and nonlinear synchronisation occur while the patients remain vacant during their epileptic absences. This type of epilepsy is an ideal example of widely distributed linear ictal neuronal synchronisation, during which deep transient loss of consciousness occurs; the patients remain alert but deeply unresponsive. Ictal EEG data analysis shows widely distributed very high levels of linear synchronisation while cross frequency nonlinear interactions, involving high gamma and beta frequencies before the epileptic seizures, precipitate during the short lived paroxysmal epileptic attacks and reappear immediately after the cessation of the seizures. At the same time linear synchronisation levels disintegrate and normal cognitive function re-emerges. Although this analogy may sound far-fetched, it gives an idea of how complex and dynamic can be the linear and non-linear brain network dynamics that underpin normal cognitive functions. It also implies that linear and nonlinear dimensions of synchronisation can exhibit counterintuitive shifts in opposite directions.

This work provides the proof of principle that measuring in isolation the strength of linear synchronisation is not enough to describe the complex behaviour of brain network interactions. We provide evidence suggesting there are advantages from our approach where separate estimations of linear and nonlinear dimensions of synchronization, with their respective strength 
and dynamic variations over time as well as their spatial distributions are determined. Noticeably, the latter can be very different for linear and nonlinear estimates. This comprehensive range of features we incorporate in this novel framework offers complementary information that sheds light on hidden dimensions of brain network behaviours in healthy aging and $\mathrm{AD}$.

The high levels of non-linear synchronisation observed for participants with $\mathrm{AD}$ above the age of 70 , both for $\mathrm{EO}$ and $\mathrm{EC}$ states (Fig. 4) reveal that network dysfunction characteristics can be age related and future work in this area should consider analysing large numbers of age stratified cohorts. Our ERR method is a time domain approach, that does not provide information about the frequencies involved during those nonlinear cross-frequency interactions, which we suspect for the elderly patients with AD involve theta and delta bands, but this will be the aim of future studies.

Changes attributed to brain maturation and development like the age-related background posterior slow wave (theta and delta) EEG activity, a well-known normal finding in clinical electroencephalography, referred to as "posterior slow waves of youth" merit some attention. These slow waves can be found interspersed with normal alpha rhythms until the age of 30 [39]. This is a good example of an age-related EEG change that can be easily appreciated on visual inspection of the data by a trained physician. More sophisticated quantitative EEG analysis with fractal dimension, an approach thought to be related to the complexity of EEG signal dynamics, has demonstrated significant change in the complexity of electrical neuronal activity throughout the lifespan, with a steady increase in young and middle-aged adults followed by a fall in the elderly [40]. In addition, previous work using various EEG features and a rigorous ML framework [36], estimated fairly reliably the participants chronological age exclusively based on brain electrical recordings. In this work we show that for linear synchronisation, HC participants below the age of 70 have clear differences in the strength of synchronisation between EO and EC states, stronger and more widespread in the posterior quadrant brain areas in the latter state. This network reactivity to eye opening is lost in the elderly $\mathrm{HC}$ group. In addition, the nonlinear estimates of DRC show higher variability in synchronisation with relatively wider spatial distribution only in the younger group during EO state. Of note, estimates of nonlinear functional connectivity show very little variation between EO and EC states in the elderly in the HC cohort. Equally, the strength of nonlinear synchronisation with AMM, remains consistent for EO and EC states both for the younger and older healthy participants. This observation suggests that this nonlinear approach produces state independent resting state EEG estimates of brain functional connectivity and that it could be applied on large EEG databases where the EO versus EC state is not known and data selection can easily become automated.

\section{CONCLUSIONS}

This paper proposed a novel brain functional connectivity imaging technology, particularly aiming to determine the contribution of nonlinearity and dynamics, on distinguishing participants with AD from HC. The parametric method used in this work is established upon a NFIR model, and a revised orthogonal least square algorithm is proposed to estimate the linear, nonlinear and combined connectivity between any two EEG channels. This approach, where linear and non-linear associations and their spatial distribution and dynamics can be estimated independently, offered us the means to dissect the dynamic brain network disruption in $\mathrm{AD}$ from another angle and to get some insight into the effect of age in HCs. This algorithm does not require to establish an unbiased full model, which reduces the dependency on the number of sampling and model validation, which is attractive to understand a highly unknown complex system. A new parameter, called Dynamic Range Connectivity, is introduced to represent the dynamics of functional connectivity. A new functional connectivity visualisation method, the RCG, is also proposed to offer a visualisable conception and representation of linear and nonlinear associations and their dynamic changes between different brain regions during EO and EC states.

Although the number of participants in this study is small, the spatial distribution and the linear and nonlinear dynamic behaviour of network disruption, revealed with the aforementioned approaches, gave us a glimpse into the complexity of brain network behaviours and spatial characteristics in health and AD, for participants younger and older than 70. Although this paper focuses on the application of this novel methodology on dementia, the developed approach is generic and can act as a powerful tool to better understand brain degeneration or dysfunction in a user friendly and systematic way.

\section{REFERENCES}

[1] M. Pievani, W. de Haan, T. Wu, W. W. Seeley, and G. B. Frisoni, "Functional network disruption in the degenerative dementias," Lancet Neurol., vol. 10, no. 9, pp. 829-843, Sep. 2011.

[2] F. Varela, J.-P. Lachaux, E. Rodriguez, and J. Martinerie, “The brainweb: Phase synchronization and large-scale integration," Nat. Rev. Neurosci., vol. 2, no. 4, pp. 229-239, Apr. 2001.

[3] M. D. Fox and M. E. Raichle, "Spontaneous fluctuations in brain activity observed with functional magnetic resonance imaging," Nat. Rev. Neurosci., vol. 8, no. 9, pp. 700-711, Sep. 2007.

[4] G. Lioi, S. L. Bell, D. C. Smith, and D. M. Simpson, "Directional connectivity in the EEG is able to discriminate wakefulness from NREM sleep," Physiol. Meas., vol. 38, no. 9, pp. 1802-1820, Aug. 2017.

[5] G. Krishna Dash, C. Rathore, M. K. Jeyaraj, P. Wattamwar, S. P. Sarma, K. Radhakrishnan, and R. Madhavan, "Interictal regional paroxysmal fast activity on scalp EEG is common in patients with underlying gliosis," 2018.

[6] R. Renzel, C. R. Baumann, I. Mothersill, and R. Poryazova, "Persistent generalized periodic discharges: A specific marker of fatal outcome in cerebral hypoxia," 2017.

[7] H. Watanabe, K. Terada, N. Suzuki, M. Ishisaka, Y. Naitoh, R. Ishihara, H. Shimoeda, T. Konagaya, and Y. Inoue, "P1-3-10. Effect of hyperventilation on seizures and EEG findings during routine EEG," Clin. Neurophysiol., vol. 129, no. 5, p. e38, May 2018.

[8] E. Visani, G. Varotto, S. Binelli, L. Fratello, S. Franceschetti, G. Avanzini, and F. Panzica, "Photosensitive epilepsy: Spectral and coherence analyses of EEG using $14 \mathrm{~Hz}$ intermittent photic stimulation," 2010.

[9] D. Blackburn, Y. Zhao, M. De Marco, S. Bell, F. He, H.-L. Wei, S. Lawrence, Z. Unwin, M. Blyth, J. Angel, K. Baster, T. Farrow, I. Wilkinson, S. Billings, A. Venneri, and P. Sarrigiannis, "A Pilot Study Investigating a Novel Non-Linear Measure of Eyes Open 
versus Eyes Closed EEG Synchronization in People with Alzheimer's Disease and Healthy Controls," Brain Sci., vol. 8, no. 7, p. 134, Jul. 2018

[10] P. G. Sarrigiannis, Y. Zhao, F. He, S. A. Billings, K. Baster, C. Rittey, J. Yianni, P. Zis, H. Wei, M. Hadjivassiliou, and R. Grünewald, "The cortical focus in childhood absence epilepsy; evidence from nonlinear analysis of scalp EEG recordings," Clin. Neurophysiol., vol. 129, no. 3, pp. 602-617, 2018.

[11] P. G. Sarrigiannis, Y. Zhao, H.-L. Wei, S. a Billings, J. Fotheringham, and M. Hadjivassiliou, "Quantitative EEG analysis using error reduction ratio-causality test; validation on simulated and real EEG data," Clin. Neurophysiol., vol. 125, no. 1, pp. 32-46, Jan. 2014

[12] M. Rubinov and O. Sporns, "Complex network measures of brain connectivity: Uses and interpretations," Neuroimage, vol. 52, no. 3, pp. 1059-1069, Sep. 2010.

[13] D. J. Watts and S. H. Strogatz, "Collective dynamics of "smallworld' networks," Nature, vol. 393, no. 6684, pp. 440-442, Jun. 1998.

[14] O. Sporns, C. J. Honey, and R. Kötter, "Identification and Classification of Hubs in Brain Networks," PLoS One, vol. 2, no. 10, p. e1049, Oct. 2007.

[15] D. Rivière, J.-F. Mangin, D. Papadopoulos-Orfanos, J.-M. Martinez V. Frouin, and J. Régis, "Automatic recognition of cortical sulci of the human brain using a congregation of neural networks," Med. Image Anal., vol. 6, no. 2, pp. 77-92, Jun. 2002.

[16] G. Lohmann, D. S. Margulies, A. Horstmann, B. Pleger, J. Lepsien, D. Goldhahn, H. Schloegl, M. Stumvoll, A. Villringer, and R.

Turner, "Eigenvector Centrality Mapping for Analyzing Connectivity Patterns in fMRI Data of the Human Brain," PLoS One, vol. 5, no. 4, p. e10232, Apr. 2010.

[17] C. J. Stam, E. C. W. Van Straaten, E. Van Dellen, P. Tewarie, G. Gong, A. Hillebrand, J. Meier, and P. Van Mieghem, "The relation between structural and functional connectivity patterns in complex brain networks," 2016.

[18] M.-E. Lynall, D. S. Bassett, R. Kerwin, P. J. McKenna, M. Kitzbichler, U. Muller, and E. Bullmore, "Functional connectivity and brain networks in schizophrenia.," J. Neurosci., vol. 30, no. 28, pp. 9477-87, Jul. 2010.

[19] S. J. Kiebel, M. I. Garrido, R. Moran, C.-C. Chen, and K. J. Friston, "Dynamic causal modeling for EEG and MEG," Hum. Brain Mapp., vol. 30, no. 6, pp. 1866-1876, Jun. 2009.

[20] M. D. Greicius and D. L. Kimmel, "Neuroimaging insights into network-based neurodegeneration," Curr. Opin. Neurol., vol. 25, no. 6, pp. 727-734, Dec. 2012

[21] J. A. S. Kelso, Understanding Complex Systems. Heidelberg: Springe, 2005.

[22] K. Lehnertz, "Epilepsy and Nonlinear Dynamics," J. Biol. Phys. vol. 34, no. 3-4, pp. 253-266, Aug. 2008.

[23] A. K. Seth, A. B. Barrett, and L. Barnett, "Granger Causality Analysis in Neuroscience and Neuroimaging," J. Neurosci., vol. 35, no. 8, pp. 3293-3297, Feb. 2015.

[24] Y. Zhao, E. Hanna, G. R. Bigg, and Y. Zhao, "Tracking Nonlinear Correlation for Complex Dynamic Systems Using a Windowed Error Reduction Ratio Method," Complexity, vol. 2017, p. 8570720 , 2017.

[25] D. Abásolo, R. Hornero, P. Espino, J. Poza, C. I. Sánchez, and R. de la Rosa, "Analysis of regularity in the EEG background activity of Alzheimer's disease patients with Approximate Entropy," Clin. Neurophysiol., vol. 116, no. 8, pp. 1826-1834, Aug. 2005.
[26] R. McMackin, M. Muthuraman, S. Groppa, C. Babiloni, J.-P. Taylor, M. C. Kiernan, B. Nasseroleslami, and O. Hardiman, "Measuring network disruption in neurodegenerative diseases: New approaches using signal analysis," J. Neurol. Neurosurg. Psychiatry, p. jnnp-2018-319581, Feb. 2019.

[27] R. Guerreiro and J. Bras, "The age factor in Alzheimer's disease," Genome Med., vol. 7, no. 1, p. 106, Dec. 2015.

[28] M. Petti, J. Toppi, F. Babiloni, F. Cincotti, D. Mattia, and L. Astolfi, "EEG Resting-State Brain Topological Reorganization as a Function of Age," Comput. Intell. Neurosci., vol. 2016, pp. 1-10, 2016.

[29] R. Ishii, L. Canuet, Y. Aoki, M. Hata, M. Iwase, S. Ikeda, K. Nishida, and M. Ikeda, "Healthy and Pathological Brain Aging: From the Perspective of Oscillations, Functional Connectivity, and Signal Complexity," Neuropsychobiology, vol. 75, no. 4, pp. 151$161,2017$.

[30] C. S. Musaeus, K. Engedal, P. Høgh, V. Jelic, M. Mørup, M. Naik, A.-R. Oeksengaard, J. Snaedal, L.-O. Wahlund, G. Waldemar, and B. B. Andersen, "Oscillatory connectivity as a diagnostic marker of dementia due to Alzheimer's disease," Clin. Neurophysiol., vol. 130, no. 10, pp. 1889-1899, Oct. 2019.

[31] W. de Haan, Y. AL Pijnenburg, R. L. Strijers, Y. van der Made, W. M. van der Flier, P. Scheltens, and C. J. Stam, "Functional neural network analysis in frontotemporal dementia and Alzheimer's disease using EEG and graph theory," BMC Neurosci., vol. 10, no. 1, p. 101, Dec. 2009.

[32] B. Dubois, H. H. Feldman, C. Jacova, S. T. DeKosky, P. BarbergerGateau, J. Cummings, A. Delacourte, D. Galasko, S. Gauthier, G. Jicha, K. Meguro, J. O’Brien, F. Pasquier, P. Robert, M. Rossor, S. Salloway, Y. Stern, P. J. Visser, and P. Scheltens, "Research criteria for the diagnosis of Alzheimer's disease: revising the NINCDSADRDA criteria," Lancet Neurol., vol. 6, no. 8, pp. 734-746, Aug. 2007.

[33] S. A. Billings, Nonlinear System Identification. Chichester, UK: John Wiley \& Sons, Ltd, 2013.

[34] Y. Zhao, S. A. Billings, H. Wei, and P. G. Sarrigiannis, "Tracking time-varying causality and directionality of information flow using an error reduction ratio test with applications to electroencephalography data," Phys. Rev. E, vol. 86, no. 5, p. 051919, Nov. 2012.

[35] P. Kassebaum, "circularGraph - File Exchange - MATLAB Central," 2016.

[36] P. Durongbhan, Y. Zhao, L. Chen, P. Zis, M. De Marco, Z. C. Unwin, A. Venneri, X. He, S. Li, Y. Zhao, D. J. Blackburn, and P. G. Sarrigiannis, "A Dementia Classification Framework Using Frequency and Time-Frequency Features Based on EEG Signals," IEEE Trans. Neural Syst. Rehabil. Eng., vol. 27, no. 5, pp. 826-835, May 2019.

[37] M. G. Preti, T. Aw Bolton, and D. Van De Ville, "The dynamic functional connectome: State-of-the-art and perspectives," 2017.

[38] A. Schnitzler and J. Gross, "Normal and pathological oscillatory communication in the brain," Nat. Rev. Neurosci., vol. 6, no. 4, pp. 285-296, Apr. 2005.

[39] E. Niedermeyer and F. L. Da Silva., Electroencephalography. 530 Walnut street, Philadelphia, PA 19106, USA: Lippincott Williams \& Wilkins, 2005.

[40] F. Zappasodi, L. Marzetti, E. Olejarczyk, F. Tecchio, and V. Pizzella, "Age-Related Changes in Electroencephalographic Signal Complexity," PLoS One, vol. 10, no. 11, p. e0141995, Nov. 2015. 
2019-11-14

\section{Imaging of nonlinear and dynamic}

functional brain connectivity based on

EEG recordings with the application on

the diagnosis of Alzheimer's disease

\section{Zhao, Yifan}

\section{IEEE}

Zhao $\mathrm{Y}$, Zhao $\mathrm{Y}$, Durongbhan $\mathrm{P}$, et al., Imaging of nonlinear and dynamic functional brain connectivity based on EEG recordings with the application on the diagnosis of Alzheimer's disease. IEEE Transactions on Medical Imaging, Available online 14 November 2019 https://doi.org/10.1109/TMI.2019.2953584

Downloaded from Cranfield Library Services E-Repository 